\title{
Exact solution of earth-flattening transfor- mation for P-SV waves: Taking surface wave as an example*
}

\author{
HUANG Hui (黄 慧) CHEN Xiao-fei ${ }^{*}$ (陈晓非) \\ Computational Geodynamics Laboratory, School of Earth and Space Sciences, Peking University, Beijing 100871, China
}

\begin{abstract}
Taking surface wave as an example this paper proposes an exact solution of earth-flattening transformation for P-SV waves and discusses the applicability of the approximate methods. The results show that the transform parameter $m$ has little influence on the final results, and on the condition of short wave approximation, approximate earth-flattening transformation is suitable. Moreover, the efficiency of approximate transformation is twice of that of exact transformation. For low frequency problems exact earth-flattening transformation should be used.
\end{abstract}

Key words: earth-flattening transformation; generalized reflection-transmission coefficients method; eigen-displacement and traction; phase velocity; surface wave

CLC number: P315. $3^{+} 1$ Document code: A

\section{Introduction}

The theory and computational method of seismic wave propagation in flat-layered medium is well studied and applied in seismology. The flat-layered medium is an approximation of the real Earth model. It is only suitable for small epicentral distance and high frequency seismic problems. For large epicentral distance and low frequency problems the curvature of interfaces cannot be ignored. We should solve these problems in spherical coordinates in which the calculation is much more complicated and harder than that in Cartesian coordinates. Earth-flattening transformation (Biswas, Knopoff, 1970; Biswas, 1972; Bhattacharya, 1996; Dahlen, Tromp, 1998) is able to convert seismic wave equations in spherical coordinates into Cartesian coordinates to simplify the calculation. For SH wave Biswas and Knopoff (1970) proposed an exact transformation formula. But for P-SV waves because of the coupling of P and SV waves, the terms of elastodynamic equation in spherical and Cartesian coordinates do not correspond to each other. The difference is inversely proportional to the radius of the Earth. Biswas (1972) ignored these high-order terms to get an approximate earth-flattening transformation for P-SV waves. Taking surface wave for an example Bhattacharya (1996) solved the transformed equations using differential equation theory. But the process is complicated and easy to omit the fundamental phase velocity. Bhattacharya and Arora (1997) extended Bhattacharya's method (1996) to transverse isotropic media. Using earth-flattening transformation Bhattacharya (2005) calculated synthetic seismogram for spherical

\footnotetext{
* Received 2007-06-05; accepted in revised form 2007-06-28.

Foundation item: National Natural Science Foundation of China (40474011, 40521002).

^ Author for correspondence: xfchen@pku.edu.cn
} 
layered Earth model.

This paper proposes a direct and exact method of transformed equations for P-SV waves based on generalized reflection-transmission coefficients method (Chen, 1993, 1999) for flat-layered Earth models. And the efficiency and applicability of the approximate earth-flattening method are also discussed.

\section{Basic equation and earth-flattening transformation}

The normal mode of seismic surface wave and its frequency dispersion satisfy the elastodynamic equations without source term (Chen, 1993). For seismic surface wave the gravity field of the Earth can be ignored (Aki, Richards, 1980). In frequency domain, the elastodynamic equations of a non-gravitating spherical symmetric, non-rotating perfectly elastic and isotropic (SNREI) Earth model without source term can be written as following (Dahlen, Tromp, 1998):

$$
\begin{aligned}
& r^{-2} \frac{\mathrm{d}}{\mathrm{d} r}\left[r^{2}(\lambda+2 \mu) \frac{\mathrm{d} U}{\mathrm{~d} r}+\lambda r(2 U-\sqrt{N} V)\right]+r^{-1}\left[(\lambda+2 \mu) \frac{\mathrm{d} U}{\mathrm{~d} r}+\lambda r^{-1}(2 U-\sqrt{N} V)\right]- \\
& (3 \lambda+2 \mu) r^{-1}\left(\frac{\mathrm{d} U}{\mathrm{~d} r}+2 r^{-1} U-\sqrt{N} r^{-1} V\right)-\sqrt{N} \mu r^{-1}\left(\frac{\mathrm{d} V}{\mathrm{~d} r}-r^{-1} V+\sqrt{N} r^{-1} U\right)+\omega^{2} \rho U=0 \\
& r^{-2} \frac{\mathrm{d}}{\mathrm{d} r}\left[\mu r^{2}\left(\frac{\mathrm{d} V}{\mathrm{~d} r}-r^{-1} V+\sqrt{N} r^{-1} U\right)\right]+\mu r^{-1}\left(\frac{\mathrm{d} V}{\mathrm{~d} r}-r^{-1} V+\sqrt{N} r^{-1} U\right)+\sqrt{N} \lambda r^{-1} \frac{\mathrm{d} U}{\mathrm{~d} r}+ \\
& \sqrt{N}(\lambda+\mu) r^{-2}(2 U-\sqrt{N} V)+\left[\omega^{2} \rho-(N-2) \mu r^{-2}\right] V=0
\end{aligned}
$$

where $U$ and $V$ are the two components of displacement field for P-SV waves (i.e. spherical oscillation) under spherical harmonic basis and are only functions of the radius $r ; N=\sqrt{l(l+1)}$ where $l(0 \leq l \leq \infty)$ is the spherical harmonic order; $\rho, \lambda, \mu$ are density and Lame constants respectively. For spherically symmetric Earth model P-SV and SH waves are independent of each other. The process of solving SH problem is similar to P-SV problem but simpler. So, we only discuss P-SV waves in this paper.

The two traction components on an arbitrary spherical surface under spherical harmonic basis are (Dahlen, Tromp, 1998):

$$
\begin{gathered}
R=(\lambda+2 \mu) \frac{\mathrm{d} U}{\mathrm{~d} r}+\lambda r^{-1}(2 U-\sqrt{N} V) \\
S=\mu\left(\frac{\mathrm{d} V}{\mathrm{~d} r}-r^{-1} V+\sqrt{N} r^{-1} U\right)
\end{gathered}
$$

Correspondingly, the zero-traction boundary condition on the surface of the Earth is

$$
\left.R\right|_{r=a}=\left.S\right|_{r=a}=0
$$

and the displacement and traction continuities condition in the interior's interfaces are

$$
[U]_{-}^{+}=[V]_{-}^{+}=[R]_{-}^{+}=[S]_{-}^{+}=0
$$

where []$_{-}^{+}$means the difference of a function value above and beneath a certain interface.

To simplify the basic equations (1) we introduce the following earth-flattening transformation (Biswas, 1972; Bhattacharya, 1996; Dahlen, Tromp, 1998): 


$$
\begin{aligned}
& z=a \ln \left(\frac{a}{r}\right) \quad U(r)=\left(\frac{r}{a}\right)^{m} u_{1}(z) \quad V(r)=\left(\frac{r}{a}\right)^{m} u_{2}(z) \quad k_{f}^{2}=\frac{N}{a^{2}} \\
& \lambda_{f}(z)=\left(\frac{r}{a}\right)^{m-1} \lambda(r) \quad \mu_{f}(z)=\left(\frac{r}{a}\right)^{m-1} \mu(r) \quad \rho_{f}(z)=\left(\frac{r}{a}\right)^{m+1} \rho(r)
\end{aligned}
$$

where $u_{1}$ and $u_{2}$ are transformed displacement field, subscript $f$ stands for that the parameter is in flat Earth frame, $m$ is the transform exponent and $a$ is the Earth even radius $(6371 \mathrm{~km})$. Variable $z$ gradually increases from Earth surface to core. At the surface of the Earth $z=0$ and at the center of the Earth $z \rightarrow \infty$. Through earth-flattening transformation equations (1) are converted to:

$$
\begin{gathered}
\frac{\mathrm{d}}{\mathrm{d} z} \tau_{1}(z)-\frac{\lambda_{f} k_{f}}{\lambda_{f}+2 \mu_{f}} \tau_{2}(z)-\frac{3}{a} \tau_{1}(z)+\left[\rho_{f} \omega^{2}-\frac{4 \mu_{f}\left(\lambda_{f}+\mu_{f}\right)}{\left(\lambda_{f}+2 \mu_{f}\right)} k_{f}^{2}+\frac{2 \mu_{f}}{a^{2}}\right] u_{1}(z)+ \\
\frac{2 \mu_{f}\left(3 \lambda_{f}+2 \mu_{f}\right) k_{f}}{a\left(\lambda_{f}+2 \mu_{f}\right)} u_{2}(z)=0 \\
\frac{\mathrm{d}}{\mathrm{d} z} \tau_{2}(z)+k_{f} \tau_{1}(z)-\frac{4 \mu_{f}}{a\left(\lambda_{f}+2 \mu_{f}\right)} \tau_{2}(z)+\left[\rho_{f} \omega^{2}-\frac{4 \mu_{f}\left(3 \lambda_{f}+2 \mu_{f}\right)}{a^{2}\left(\lambda_{f}+2 \mu_{f}\right)}\right] u_{2}(z)+ \\
\frac{2 \mu_{f}\left(3 \lambda_{f}+2 \mu_{f}\right) k_{f}}{a\left(\lambda_{f}+2 \mu_{f}\right)} u_{1}(z)=0
\end{gathered}
$$

The spherical traction components $(2 \mathrm{a})$ and $(2 \mathrm{~b})$ are:

$$
S(r)=-\left(\frac{a}{r}\right) r^{m} \tau_{1}(z) \text { and } R(r)=-\left(\frac{a}{r}\right) r^{m} \tau_{2}(z)
$$

where

$$
\begin{gathered}
\tau_{1}(z)=\mu_{f}\left[\frac{\mathrm{d} u_{1}}{\mathrm{~d} z}-k_{f} u_{2}(z)-\frac{(m-1)}{a} u_{1}(z)\right] \\
\tau_{2}(z)=\left(\lambda_{f}+2 \mu_{f}\right) \frac{\mathrm{d} u_{2}(z)}{\mathrm{d} z}+\lambda_{f} k_{f} u_{1}(z)-\frac{1}{a}\left[\lambda_{f}(m+2)+2 m \mu_{f}\right] u_{2}(z)
\end{gathered}
$$

Correspondingly, the transformed traction-free condition (3) and continuity conditions of the interior's interfaces (4) can be expressed as:

$$
\left.\tau_{1}(z)\right|_{z=0}=\left.0 \quad \tau_{2}(z)\right|_{z=0}=0
$$

and

$$
\left[u_{1}(z)\right]_{-}^{+}=\left[u_{2}(z)\right]_{-}^{+}=\left[\tau_{1}(z)\right]_{-}^{+}=\left[\tau_{2}(z)\right]_{-}^{+}=0
$$

There is no approximation in deducing equations (6) and (7). They include terms inversely proportional to $a$ and $a^{2}$. When the following condition

$$
k_{f} a>>1
$$

is satisfied (i.e. short wave approximation), we can ignore the high-order terms including $a^{-1}$ and $a^{-2}$. So equations (6) and (7) and boundary conditions (8) are the same as those for flat-layered 
Earth models (Aki, Richards, 1980; Kennett, 1983; Chen, 1993) and do not relate with exponent $m$. So on the condition of the short wave approximation the equations for spherically symmetric medium are converted to equations for flat-layered model. This is the traditional transformation (Biswas, 1972). Obviously, when short wave approximation condition is not satisfied, traditional earth-flattening transformation is not suitable any more. We propose a direct and exact method to solve equations (6) and (7) not ignoring $a^{-1}$ and $a^{-2}$ terms. So whether the short wave approximation condition is satisfied or not we can get exact solution for surface wave through earth-flattening transformation.

\section{Exact solution for transformed equations}

The transformed equations (6) and (7) can be expressed as follows

$$
\frac{\mathrm{d}}{\mathrm{d} z} \boldsymbol{y}(z)=\boldsymbol{A y}(z)
$$

where

$$
\boldsymbol{A}=\left[\begin{array}{cccc}
\frac{m-1}{a} & \boldsymbol{y}(z)=\left[u_{1}(z), u_{2}(z), \tau_{1}(z), \tau_{2}(z)\right]^{\mathrm{T}} & \\
\frac{-\lambda k}{\lambda+2 \mu} & \frac{1}{a}\left(m+\frac{2 \lambda}{\lambda+2 \mu}\right) & 0 & \frac{1}{\lambda+2 \mu} \\
\frac{4 \mu(\lambda+\mu) k^{2}}{\lambda+2 \mu}-\rho \omega^{2}-\frac{2 \mu}{a^{2}} & -\frac{2(3 \lambda+2 \mu)}{a(\lambda+2 \mu)} \mu k & \frac{3}{a} & \frac{\lambda k}{\lambda+2 \mu} \\
-\frac{2(3 \lambda+2 \mu)}{a(\lambda+2 \mu)} \mu k & -\rho \omega^{2}+\frac{4 \mu(3 \lambda+2 \mu)}{(\lambda+2 \mu) a^{2}} & -k & \frac{4 \mu}{a(\lambda+2 \mu)}
\end{array}\right]
$$

For simplicity we ignore the subscript $f$. The traction-free and continuity conditions are

$$
\left.\boldsymbol{y}_{2}(z)\right|_{z=0}=0
$$

and

$$
[y(z)]_{-}^{+}=0
$$

The above first-order differential equations (10) of displacement-traction vector $\boldsymbol{y}(z)$ and their boundary conditions (11) is formally the same as those for flat-layered Earth model (Chen, 1993; HE et al, 2006). The only difference is the coefficient matrix $\boldsymbol{A}$. So we can follow the Chen's GRT method (1993) to solve them.

Flat-layered model is the optimal approximation to the flat Earth model. We solve the problem under the frame of flat-layered model. According Chen (1993) the solution of the equation set (10) can be written as

$$
\boldsymbol{y}^{(j)}(z)=\boldsymbol{E}^{(j)} \Lambda^{(j)}(z) \boldsymbol{C}^{(j)} \quad j=1,2,3, \cdots, N, N+1
$$

where $j$ is the number of the layer and there are $N+1$ layers in all; $\boldsymbol{C}^{(j)}$ is the coefficient to be determined; $\boldsymbol{E}^{(j)}$ and $\boldsymbol{\Lambda}^{(j)}$ are got in the following way:

$$
\boldsymbol{A}^{(j)}=\boldsymbol{E}^{(j)} \boldsymbol{\Gamma}^{(j)} \boldsymbol{E}^{(j)-1}
$$




$$
\begin{aligned}
\boldsymbol{E}^{(j)} & =\left[\boldsymbol{e}_{1}, \boldsymbol{e}_{2}, \boldsymbol{e}_{3}, \boldsymbol{e}_{4}\right] \\
\boldsymbol{\Gamma}^{(j)}(z)= & {\left[\begin{array}{cccc}
\varepsilon_{1} & 0 & 0 & 0 \\
0 & \varepsilon_{2} & 0 & 0 \\
0 & 0 & \varepsilon_{3} & 0 \\
0 & 0 & 0 & \varepsilon_{4}
\end{array}\right] }
\end{aligned}
$$

and

$$
\boldsymbol{\Lambda}^{(j)}(z)=\left[\begin{array}{cccc}
e^{\varepsilon_{1} z} & 0 & 0 & 0 \\
0 & e^{\varepsilon_{2} z} & 0 & 0 \\
0 & 0 & e^{\varepsilon_{3} z} & 0 \\
0 & 0 & 0 & e^{\varepsilon_{4} z}
\end{array}\right]
$$

where $\varepsilon_{i}$ and $\boldsymbol{e}_{i}$ are the $i$-th eigen-value and eigen-vector of matrix $\boldsymbol{A}^{(j)}$ respectively. $\mathcal{E}_{i}$ is determined by $\operatorname{det}\left\{\varepsilon_{i} \boldsymbol{I}-\boldsymbol{A}\right\}=0$, which is a 4 th order algebraic equation and the detailed expression of its solution can refer to YE and SHEN (2006). After $\mathcal{E}_{i}$ is solved, we can get $\boldsymbol{e}_{i}$ from $\boldsymbol{A} \boldsymbol{e}_{i}=\mathcal{E}_{i} \boldsymbol{e}_{i}$.

To calculate $\boldsymbol{C}^{(j)}$ efficiently we use generalized reflection-transmission coefficients method (Chen, 1993; HE et al, 2006). As we mentioned before, when ignoring the high-order terms the coefficient matrix $\boldsymbol{A}$ is regressed into $\boldsymbol{A}_{0}$ :

$$
A_{0}=\left[\begin{array}{cccc}
0 & k & \frac{1}{\mu} & 0 \\
\frac{-\lambda k}{\lambda+2 \mu} & 0 & 0 & \frac{1}{\lambda+2 \mu} \\
\frac{4 \mu(\lambda+\mu) k^{2}}{\lambda+2 \mu}-\rho \omega^{2} & 0 & 0 & \frac{\lambda k}{\lambda+2 \mu} \\
0 & -\omega^{2} \rho & -k & 0
\end{array}\right]
$$

It is the same as the coefficient matrix for flat-layered Earth model (Chen, 1993). In this case we can use the results of Chen (1993) directly.

To examine the accuracy of our method for surface waves (phase velocity, eigendisplacement and traction) we calculate the residual of the traction at the surface. For this problem we do not have analytical solution or numerical results to compare with, so traction residual becomes the only criterion to examine the accuracy of our results. The more accurate the phase velocities are, the smaller is

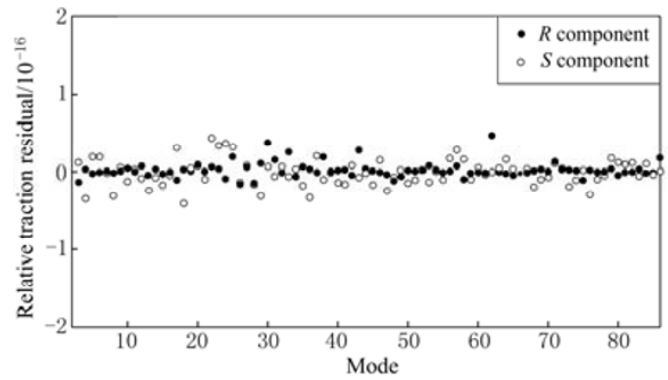

Figure 1 Relative traction residuals at free surface $(f=0.1 \mathrm{~Hz}, m=-2)$

There are 86 normal modes in all the traction residual at the surface of the Earth. Figure 1 shows the relative traction residual (the ratio of traction at the surface to the maximum traction) for Gutenberg-Bullen A spherical model (Biswas, 1972) at $f=0.1 \mathrm{~Hz}$ and $m=-2$. There are 86 normal modes in all, and the $x$-axis is their sequence number. The tolerance in searching phase velocity is $10^{-6}$ and the relative traction residuals are less than $10^{-15}$. When $m$ equals $-6,-3,0,3,6$ we get similar results. These results 
prove the accuracy of this new method to some extent.

\section{Comparison of approximate and exact transforms}

Using the exact transformation method proposed in this paper we can evaluate the accuracy of the traditional approximate transformation. Figure 2 is the first eight roots ( 86 roots in all) of the secular function of the exact transformation for the same medium model as Figure 1 at $f=0.1$ $\mathrm{Hz}$ and $m=-2$. Figure $2 \mathrm{a}$ is our results and Figure $2 \mathrm{~b}$ is that from Bhattacharya (1996). According to Chen (1993) the roots (the dark dots in the figure) are the phase velocities of surface waves. Notice that $c=3.555 \mathrm{~km} / \mathrm{s}$ (the ellipse in the figure) is $\mathrm{S}$ wave velocity of the first layer rather than the phase velocity of the fundamental mode. $c=3.267 \mathrm{~km} / \mathrm{s}$ (the dark rectangle in the figure) corresponds to discontinuity point of the real part of the secular function and is not a phase velocity either. The fundamental mode velocity is $c=3.337 \mathrm{~km} / \mathrm{s}$. Except the fundamental phase velocity our results are the same as Bhattacharya's. Bhattacharya took the first layer S wave velocity $c=3.555 \mathrm{~km} / \mathrm{s}$ (corresponding to the branch point of the secular function) as the fundamental phase velocity, but omitted the true root $c=3.337 \mathrm{~km} / \mathrm{s}$. To solve this "omitted fundamental phase velocity" Chen (1993) and HE et al (2006) proposed fundamental frequency dispersion equation in generalized reflection-transmission coefficients method to find the fundamental root fast and correctly.
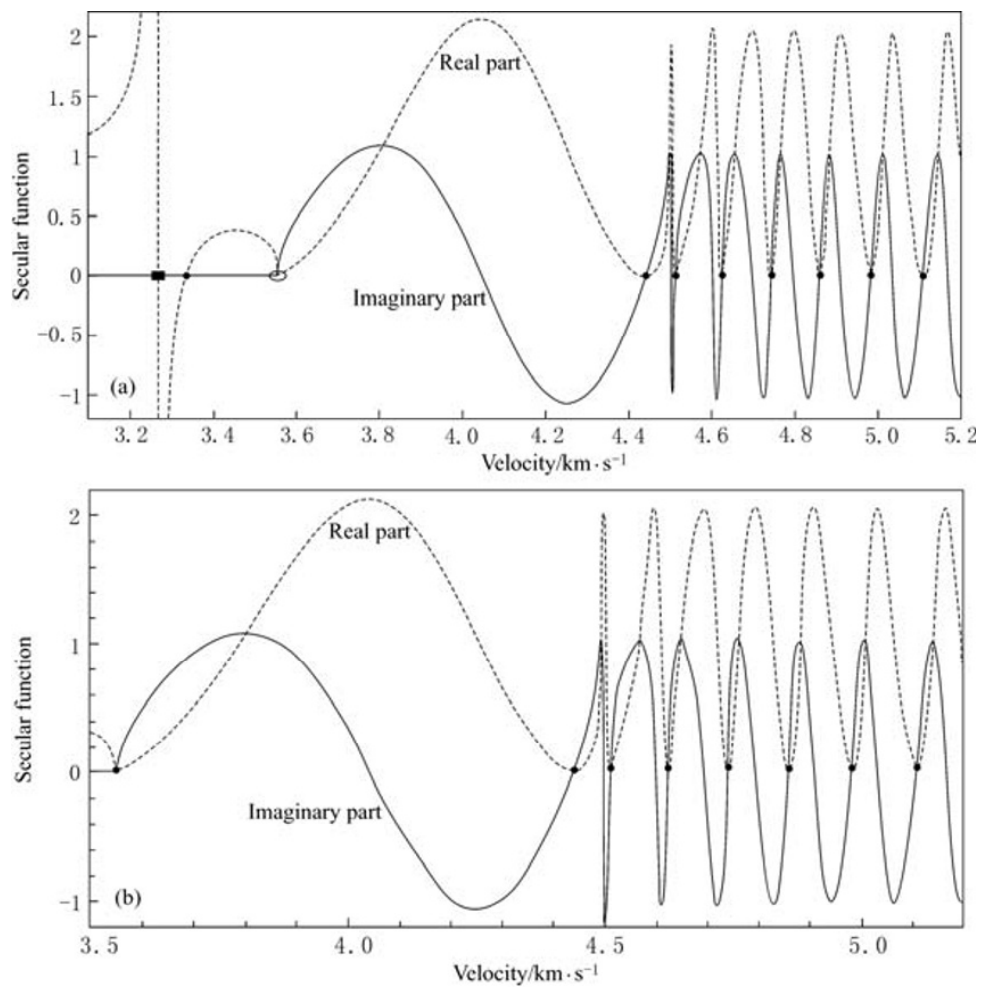

Figure 2 The first secular function of the exact transformation for Gutenberg-Bullen A spherical model at $f=0.1 \mathrm{~Hz}$ and $m=-2$

The dark dots are the first 8 phase velocities. The dark rectangle and ellipse correspond to discontinuity point of the real part and $\mathrm{S}$ wave velocity of the first layer

(a) This study's result; (b) The result from Bhattacharya (1996) 
Figure 3 gives the $m$ 's influence on the phase velocities from the 1 st to the 9 th order. When $m$ varies from -6 to 6 the phase velocities change with a magnitude of $10^{-3}$. Figure 4 shows eigen-displacement $U$ and traction $R$ of the 5 th mode. When $m$ changes from -6 to 6 the relative changes are less than $10^{-2}$. These results show though different $m$ corresponds to different transformed medium structure [formula (5)], the final results are very close. This is because $m$ is merely a parameter in transformation which is introduced to simplify the calculation. We choose $m=-2$ in following calculation.

Figure 5 and Figure 6 compare the

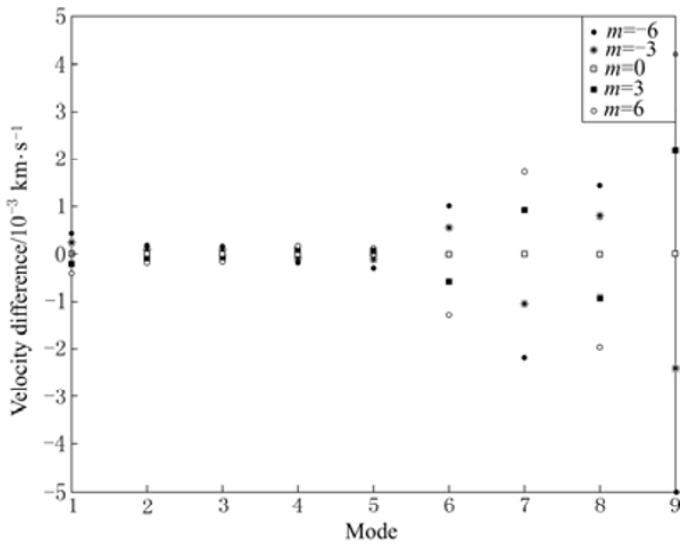

Figure $3 \mathrm{~m}$ 's influence on phase velocity from the 1 st to 9 th order (i.e. the 2 nd to 10 th root) The $x$-axis is the order and $y$-axis is the difference of phase velocity between when $m=0$ and other values results of exact transformation with approximate transformation. Figure 5 shows the difference of the phase velocities with a range of $10^{-3}$. Figure 6 compares horizontal eigen-displacement and traction of the 5 th mode phase velocities. The difference is in the magnitude of $10^{-2}$. In the coefficient matrix $\boldsymbol{A}$ of elastodynamic equation the ratios of high order terms to terms in the corresponding positions (i.e. the ratios of $\boldsymbol{A}_{12}$ and $\boldsymbol{A}_{21}$ to $\boldsymbol{A}_{11}$ and $\left.\boldsymbol{A}_{22}\right)$ are about $1 /(\mathrm{ka})$. So we can use $k a$ to evaluate the extent of approximation. Here we have $k a=\omega a / c \sim 80(f=0.01 \mathrm{~Hz}, c(\omega) \approx 5.0 \mathrm{~km} / \mathrm{s})$ which roughly satisfies the short wave
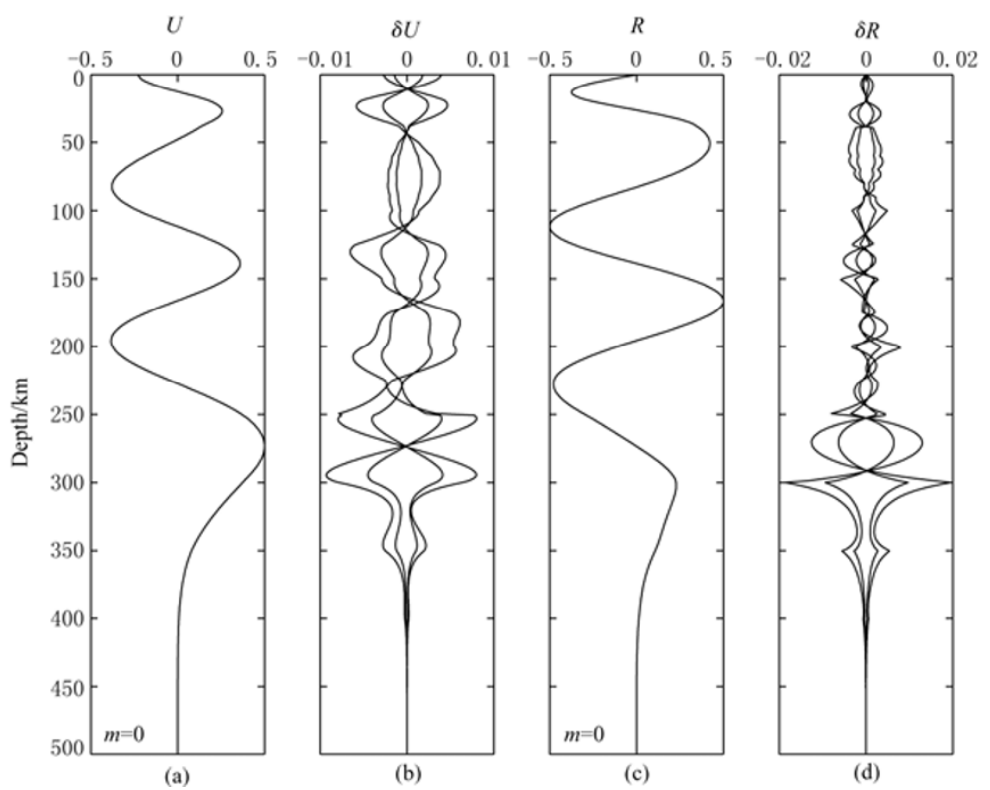

Figure 4 (a) and (c) are eigen-displacement $U$ and traction $R$ of the 5 th mode when $m=0$, they have both been normalized with 0.5 as the maximum absolute value; (b) and (d) are the differences of displacement and traction between $m=-6,-3,3,6$ and $m=0$ 


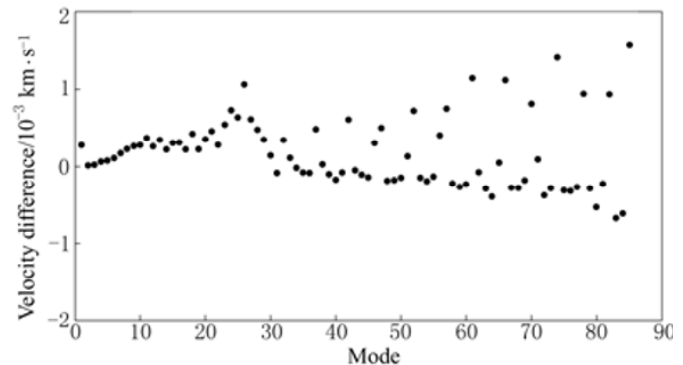

Figure 5 Difference of phase velocity between exact and approximate earth-flattening transformation approximation condition $k a>>1$. Taking the fundamental phase velocity for example Figure 7 shows the comparison of phase velocity between exact and approximate transformation. When $f>0.004 \mathrm{~Hz}$ (period $T<250 \mathrm{~s}$ ), $k a>30$ and the relative error of phase velocity by approximate method is less than $5 \%$. So when frequency is larger than $0.004 \mathrm{~Hz}$ (period $T<250 \mathrm{~s}$ ) the traditional approximate earth-flattening transformation is suitable. Furthermore, the calculation efficiency of approximate transformation is higher than exact transformation. Figure 8 compares the calculation efficiency of approximate transformation with exact transformation. The consumed time by exact transformation is about twice of the approximate method. The difference between exact and approximate transformation in computation efficiency lies in the calculation of matrix $\boldsymbol{E}$. In approximate transformation $\boldsymbol{E}_{0}$ corresponding to $\boldsymbol{A}_{0}$ has analytical expression (Chen, 1993), while $\boldsymbol{E}$ in exact transformation is calculated from one 4-order algebraic equation and 4 linear equations. Because the computation time of $k a$ and $\boldsymbol{E}$ are not related to parameters of medium, so the above two conclusions do not change for different media.

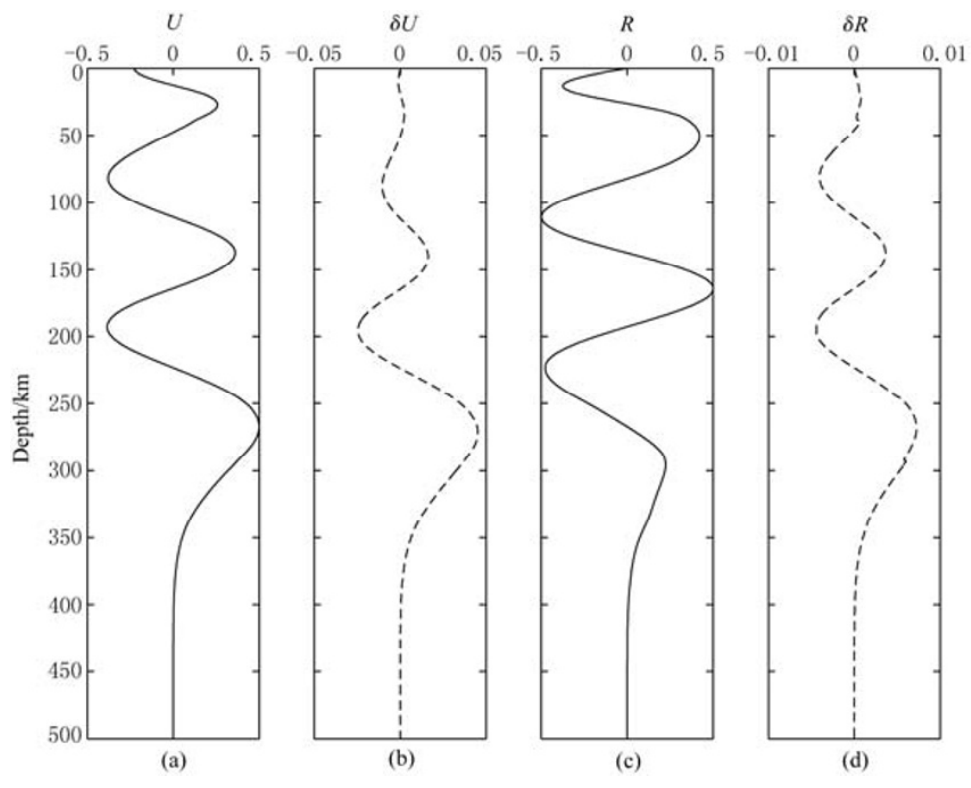

Figure 6 (a) and (c) are the 5th mode's exact eigen-displacement $U$ and traction $R$ when $m=0$; (b) and (d) are the differences between approximate and exact transformation when $m=0$

\section{Conclusions}

In the present paper we deduce the exact transformed equations of a non-gravitating SNREI Earth model without source term and give the numerical results for seismic surface wave using 


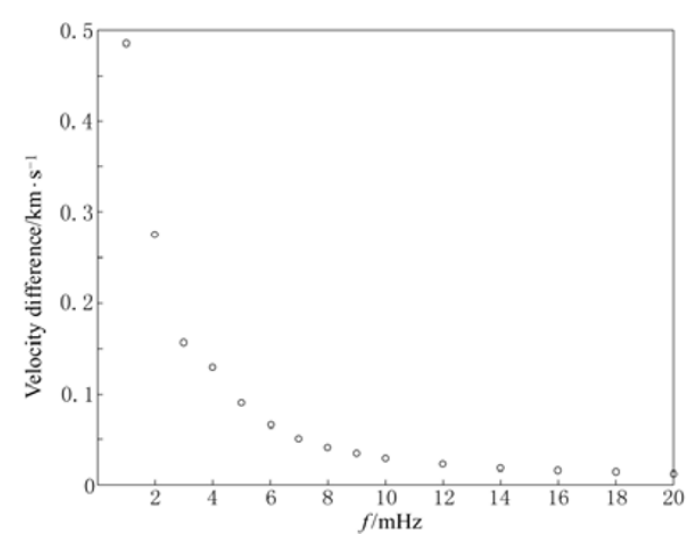

Figure 7 The comparison of phase velocity between exact and approximate transformation (taking the example the fundamental phase velocity for example)

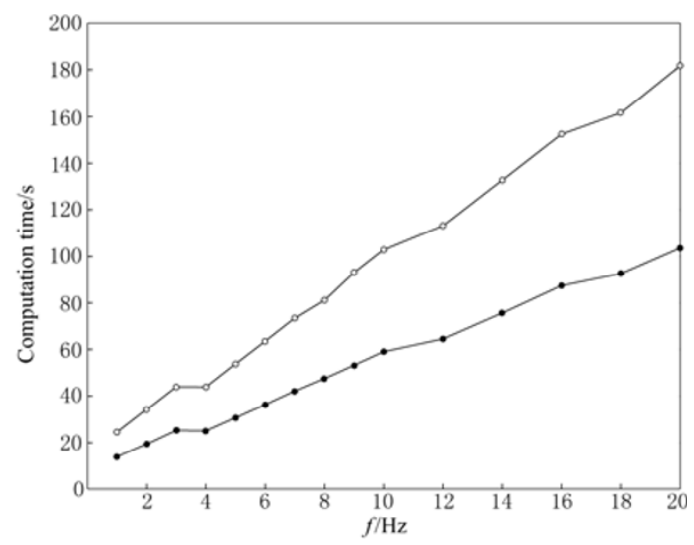

Figure 8 Computation efficiency: The time consumed in searching roots of exact (denoted by circles) and approximate (denoted by dots) transformations

generalized reflection-transmission coefficients method. Upon this basis we also discuss the efficiency and applicability of the approximate earth-flattening method. The following are our conclusions:

1) The transform exponent $m$ has little influence on the final results so we can choose an arbitrary value. In this paper we choose $m=-2$.

2) On the condition of short wave approximation the approximate earth-flattening transformation is suitable; when $k a>30$ (i.e. $f>0.004 \mathrm{~Hz}$ or $T<250 \mathrm{~s}$ ) the error of approximate method is less than $5 \%$.

3) The efficiency of the approximate transformation is twice of the exact transformation.

So when the short wave approximation condition is satisfied we should use approximate earth-flattening transformation. In this case both accuracy and efficiency are ensured. But for low frequency problem we should use exact earth-flattening transformation. The exact transformation and the comments on approximate transformation in this paper are not only suitable for seismic surface wave but also for body wave and synthetic seismogram of spherical Earth model.

\section{References}

Aki K, Richards P G. 1980. Quantitative Seismology: Theory and Methods [M]. San Francisco: W H Freeman: 267-286. Bhattacharya S N. 1996. Short notes: Earth-flattening transformation for P-SV waves [J]. Bull Seism Soc Amer, 86: $1979-1982$.

Bhattacharya S N. 2005. Synthetic seismograms in a spherical Earth using exact flattening transformation [J]. Geophys Res Lett, 32(21): L21303.

Bhattacharya S N and Arora S. 1997. A flattening transformation for P-SV waves in a transversely isotropic earth [J]. Bull Seism Soc Amer, 87: $1297-1304$.

Biswas N. 1972. Earth-flattening procedure for the propagation of Rayleigh wave [J]. Pure Appl Geophys, 96: 61-74.

Biswas N and Knopoff L. 1970. Exact-earth-flattening calculation for Love waves [J]. Bull Seism Soc Amer, 60: $1123-1137$.

Chen X F. 1993. A systematic and efficient method of computing normal modes for multi-layered half space [J]. Geophys $J$ Int, 115: 391-409.

Chen X F. 1999. Seismograms synthesis in multi-layered half-space (I): Theoretical formulation [J]. Earthquake Research in China, 13(2): 149-174.

Dahlen F A, Tromp J. 1998. Theoretical Global Seismology [M]. Princeton: Princeton University Press: 268-272.

HE Yao-feng, CHEN Wei-tian, CHEN Xiao-fei. 2006. Normal mode computation by the generalized reflection-transmission coefficient method in planar layered half space [J]. Chinese J Geophys, 49(4): 1 074-1 081 (in Chinese).

Kennett B L N. 1983. Seismic Wave Propagation in Stratified Media [M]. New York: Cambridge University Press: 25-44.

YE Qi-xiao and SHEN Yong-huan. 2006. Mathematical Handbook [M]. Beijing: Science Press: 16-18 (in Chinese). 